\title{
Educação em Saúde para a transformação de práticas sociais, alimentares e
}

\section{nutricionais}

\author{
Health education for the transformation of social, food and nutritional practices \\ Educación sanitaria para la transformación de prácticas sociales, alimentarias y nutricionales
}

Recebido: 11/12/2021 | Revisado: 16/12/2021 | Aceito: 21/12/2021 | Publicado: 03/01/2022

Aída Couto Dinucci Bezerra

ORCID: https://orcid.org/0000-0001-9132-4390

Universidade Federal de Mato Grosso, Brasil

E-mail: aidacdbprofissional@gmail.com

Lúcia Dias da Silva Guerra

ORCID: https://orcid.org/0000-0003-0093-2687

Universidade de São Paulo, Brasil

E-mail: luciadsguerra@ alumni.usp.br

Cristianne Maria Famer Rocha

ORCID: https://orcid.org/0000-0003-3281-2911

Universidade Federal do Rio Grande do Sul

E-mail: rcristianne@gmail.com

Tomaz Mazuco Rodriguez

ORCID: https://orcid.org/0000-0003-4726-4110

Universidade de Santa Cruz do Sul, Brasil

E-mail: tmrodriguez@mx2.unisc.br

Camilo Darsie

ORCID: https://orcid.org/0000-0003-4696-000X

Universidade de Santa Cruz do Sul, Brasil

E-mail: camilodarsie@unisc.br

\begin{abstract}
Resumo
A garantia de alimentação adequada e saudável envolve práticas educacionais favoráveis à inclusão e à justiça social. Por meio do acesso a alimentos de qualidade, populações podem atingir melhores índices de saúde, fator que se alinha aos princípios sanitários e humanitários globais. Assim, pensar no apoderamento - termo que diferencia-se de empoderamento - alimentar e nutricional, por meio da articulação entre conhecimentos da Educação, da Saúde e da alimentação se torna uma ação de grande importância para os mais diversos contextos da atualidade. Portanto, o objetivo deste ensaio é destacar a possibilidade de investimento no apoderamento alimentar e nutricional, enquanto direito humano que pode ser tensionado e potencializado por meio da Educação Alimentar e Nutricional, na perspectiva das discussões que envolvem os preceitos da educação em saúde. Para tanto, opera-se com levantamento bibliográfico e documental que serve como embasamento para a discussão proposta. Entende-se, após tais movimentos, que há grandes limitações no âmbito do apoderamento alimentar, especialmente por deterioração das políticas públicas frente aos discursos econômicos. Contudo, existem boas possibilidades de implementação de ideias e ações acerca da temática que podem ajudar populações.
\end{abstract}

Palavras-chave: Alimentação; Educação em saúde; Segurança alimentar; Nutrição.

\begin{abstract}
The assurance of adequate and healthy food involves educational practices that favor inclusion and social justice. Through the access to quality food, populations can achieve better health results, a factor that matches with global health and humanitarian principles. Thus, thinking about apoderamento - a term that differs from empowerment - for food and nutrition, through the articulation between knowledge of Education, Health and food, becomes topics of great importance for the most diverse contexts. Therefore, the focus of this paper is to highlight the possibility of investing in food and nutrition apoderamento, as a human right that can be strengthened and enhanced through Food and Nutrition Education, from the perspective of discussions of health education. For this, a bibliographic and documental survey is used as a basis for the proposed discussion. It is understood, after such movements, that there are great limitations in the scope of food apoderamento, especially to the deterioration of public policies in the face of economic discourses. However, there are good possibilities for implementing ideas and actions on the subject that can help populations.
\end{abstract}

Keywords: Food; Health education; Food security; Nutrition. 


\section{Resumen}

La garantía de una alimentación adecuada y saludable implica prácticas educativas que favorezcan la inclusión y la justicia social. A través del acceso a alimentos de calidad, las poblaciones pueden lograr mejores resultados de salud, un factor que coincide con los principios humanitarios y de salud mundial. Así, pensar en el apoderamento - término que se diferencia del empoderamiento - para la alimentación y la nutrición, a través de la articulación entre conocimientos de Educación, Salud y alimentación, se convierte en temas de gran importancia para los más diversos contextos. Por tanto, el enfoque de este trabajo es resaltar la posibilidad de invertir en el apoderamento de la alimentación y la nutrición, como un derecho humano que puede ser fortalecido y potenciado a través de la Educación Alimentaria y Nutricional, desde la perspectiva de las discusiones de educación para la salud. Para ello, se utiliza un relevamiento bibliográfico y documental como base para la discusión propuesta. Se entiende, luego de tales movimientos, que existen grandes limitaciones en el alcance del apoderamento alimentario, especialmente al deterioro de las políticas públicas frente a los discursos económicos. Sin embargo, existen buenas posibilidades para implementar ideas y acciones sobre el tema que puedan ayudar a las poblaciones.

Palabras clave: Alimentación; Educación para la Salud; Seguridad alimentaria; Nutrición.

\section{Introdução}

A garantia de alimentação adequada e saudável, enquanto direito humano universal e igualitário, envolve práticas educacionais favoráveis à inclusão e à justiça social. Nesta direção, acredita-se que por meio do reconhecimento e do acesso a alimentos desejados e de qualidade, indivíduos, comunidades e/ou populações podem atingir melhores índices de saúde, fator que se alinha aos princípios sanitários e humanitários globais.

Destaca-se que a alimentação é uma necessidade básica do ser humano, não somente para fins nutricionais, mas também enquanto marcador cultural e social cujos significados variam conforme as realidades de diferentes pessoas. Os comportamentos alimentares são entendidos enquanto atos complexos que têm origem em diferentes sociedades, por meio de relações familiares, de trabalho e de lazer (Padrão et al., 2017).

No contexto das Ciências da Saúde e da Nutrição, por exemplo, vem sendo praticada uma mudança paradigmática por meio da qual o ato de se alimentar passa a ser compreendido a partir de atravessamentos culturais - globais e locais. Nessa perspectiva fortalece-se a visão de sujeitos com necessidades nutricionais associadas a desejos ligados a diversas instâncias da vida e, ainda, inseridos em distintos contextos educacionais, sociais, políticos e culturais.

Para tanto, é necessário que seja reforçada a conscientização acerca do direito de acesso à alimentação, que se associa ao enfrentamento das desigualdades econômicas, políticas e sociais que sustentam, em muitos lugares, a reprodução da pobreza, da miséria e da fome. Não é exagerado mencionar, por exemplo, as frequentes matérias jornalísticas que apresentam brasileiros, em 2021, consumindo restos de alimentos - como ossos e sobras de carne - por serem mais baratos diante de outras opções (G1, 2021; El País, 2021).

Como escreveu o dramaturgo Victor Hugo: "há sempre mais miséria entre as camadas inferiores do que fraternidade entre as superiores" (HUGO, 2021, p. 48). A partir do argumento do dramaturgo francês, ressalta-se que os Estados, ao se omitirem em suas funções de promotores dos direitos sociais, mostram-se pouco solidários com as camadas sociais mais vulneráveis no que se refere aos problemas de acesso à alimentação. Entende-se que ao protegerem práticas agrícolas e ambientais predatórias, a partir do viés econômico, passam a ser algozes no sistema alimentar, com potencial nocivo à saúde humana.

Assim, pensar no apoderamento alimentar e nutricional, por meio da articulação entre conhecimentos da Educação, da Saúde e da alimentação se torna uma ação de grande importância para os mais diversos contextos da atualidade. Portanto, o objetivo deste ensaio é destacar a necessidade de investimentos no apoderamento alimentar e nutricional, enquanto direito humano, na perspectiva das discussões que envolvem os preceitos da educação em saúde. Entende-se que há grandes limitações nesse âmbito, ao mesmo tempo em que existem boas possibilidades de implementação de ideias e ações acerca da temática. 
Partindo do exposto, nas seções que seguem, discorre-se sobre a metodologia do estudo, a alimentação é contextualizada enquanto direito humano e a educação em saúde é apresentada enquanto prática promissora para a melhoria das condições de acesso e práticas alimentares. Partindo disto, destaca-se a Educação Alimentar e Nutricional enquanto ferramenta promissora de apoderamento alimentar e nutricional.

\section{Metodologia}

Este texto se configura como um ensaio reflexivo. Deste modo, partiu da aproximação de diferentes materiais, de acordo com as necessidades dos autores, de modo a auxiliá-los no posicionamento crítico acerca da temática da educação em saúde voltada para práticas de alimentação e nutrição. Os ensaios são baseados em reflexões críticas sobre assuntos relevantes e de interesse para aqueles que os desenvolvem. Conforme destacado por Figueiredo:

o gênero textual paper ou ensaio, independentemente da nomenclatura que lhe seja atribuída, difere de um relatório, uma vez que se espera do ensaísta um juízo valorativo ou uma interpretação dos fatos ou das informações que foram recolhidas. No ensaio acadêmico, é desejável que haja o desenvolvimento de um ponto de vista acerca de um tema, uma tomada de posição definida e a expressão dos pensamentos com certa originalidade (Figueiredo, 2012, p. 6).

Partindo disto, foi desenvolvida uma reflexão crítica a partir de revisão narrativa. Rother (2007, p. 1) argumenta que "os artigos de revisão, assim como outras categorias de artigos científicos, são uma forma de pesquisa que utilizam de fontes de informações bibliográficas ou eletrônicas para obtenção de resultados de pesquisas de outros autores, com o objetivo de fundamentar teoricamente um determinado objetivo". Portanto, contou-se, aqui, com literatura científica - encontrada no Google Scholar -, a partir de revistas científicas, livros especializados e documentos disponibilizados por organizações relacionadas às temáticas da alimentação, nutrição e educação em saúde.

\section{Direito Humano à alimentação adequada}

O Relatório Global de Crises Alimentares, publicado pelo Programa Mundial de Alimentação (ONU/FAO/PMM et al, 2020), menciona que os índices de Insegurança Alimentar e Nutricional duplicaram em decorrência da pandemia de Covid-19. $\mathrm{O}$ documento também destaca que aproximadamente três bilhões de pessoas não têm condições para manterem hábitos saudáveis de alimentação. Complementarmente, o relatório da Organização pelo Direito Humano à Alimentação e à Nutrição Adequadas (FIAN, 2020), aponta que a pandemia agravou uma crise de acesso, adequação e disponibilidade de alimentos para as populações vulneráveis, em todo o mundo, devido ao aumento do desemprego e/ou à redução de renda de trabalhadores informais. No Brasil, a elevação dos preços dos alimentos, gerada, entre outros motivos, pela especulação de intermediários e pelo predomínio da exportação, tem sido intensificada pela desvalorização cambial do Real em relação ao Dólar.

Embora a problemática do consumo de alimentos não saudáveis seja anterior à pandemia, após o início da disseminação do Sars-CoV-2, a qualidade dos hábitos nutricionais piorou, já que houve uma queda drástica da média salarial, acompanhada do aumento do desemprego em escalas global e nacional. Isso direciona indivíduos e, no limite, populações, à nutrição baseada em carboidratos simples e gorduras monoinsaturadas, devido aos seus custos geralmente mais baixos, ocasionando a diminuição da qualidade de vida da população - inclusive em relação ao combate viral, por parte do sistema imunológico. Além disso, a impossibilidade de acesso a alimentos adequados causa outros problemas ligados à nutrição. A obesidade, por exemplo, aumentou 72\% nos últimos treze anos, saindo de 11,8\% em 2006 para 20,3\% em 2019 (ABESO, 2021; Unicef, 2021). De acordo com a Organização Pan-americana de Saúde : 
Fatores socioeconômicos, entre outros fatores, influenciam muitos comportamentos, inclusive hábitos alimentares. Depende muito das circunstâncias pessoais. Um indivíduo com alto nível de escolaridade, empregado, informado e com alto poder aquisitivo tem um leque muito maior de opções alimentares que um indivíduo desempregado, com baixo nível de escolaridade e baixa renda. Os pesquisadores e as organizações que dão demasiada ênfase à responsabilidade pessoal em saúde ignoram o descrito acima ao afirmarem ou implicarem que as pessoas são livres para optar por alimentos saudáveis e ter uma alimentação saudável (OPAS, 2018, p. 33).

Em alguns países latino-americanos e, em particular, no Brasil, tem ocorrido o enraizamento de práticas neoliberais, já que promove-se a redução do papel do Estado frente ao avanço do capital e da ideia de que cada sujeito é responsável pelos seus fracassos e sucessos. Destaca-se, neste contexto, que ao invés de protegerem populações vulneráveis, por meio de políticas públicas e programas sociais, diferentes governos têm ampliado o apoio às grandes cadeias agroindustriais e promovido a distribuição e o consumo de alimentos ultraprocessados, via incentivos econômicos e doação de cestas básicas pautadas em acordos comerciais.

Assim, se aniquilam condições de saúde adequadas para a maior parte da população, fazendo emergir problemas que se tornam significativos em curto, médio e longo prazos. A ideia de que o Estado está em constante crise fiscal e que deve gastar pouco com suas políticas públicas, bastante comentada na atualidade, em muitos contextos políticos, desmonta os direitos sociais (Behring \& Boschetti, 2017).

Tais práticas contrariam o Direito Humano à Alimentação Adequada, previsto na Declaração Universal dos Direitos Humanos (ONU, 1948), em seu vigésimo quinto artigo: "Todo ser humano tem direito a um padrão de vida capaz de assegurar a si e à sua família saúde, bem-estar, inclusive alimentação, vestuário, habitação, cuidados médicos e os serviços sociais indispensáveis e direito à segurança em caso de desemprego, [...]" (p. s/n). Na mesma direção, afrontam a Ação Brasileira pela Nutrição e Direitos Humanos que refere que o Direito Humano à Alimentação Adequada depende de políticas e estratégias sustentáveis de produção, de distribuição e de acesso a alimentos. Ainda, ressalta a importância da garantia de consumo de alimentos seguros e de qualidade em todos os níveis federativos (ABRANDH, 2013).

Os Direitos Humanos foram reconhecidos e consagrados ao longo da história da humanidade, sendo considerados naturais e universais por serem independentes de ato jurídico e por valerem para todos, sem fronteiras. Assim, devem ser respeitados como norma de cunho social. São, ainda, indivisíveis e interdependentes, pois não se pode aceitar separadamente direitos individuais de cunho social ou de defesa ambiental, sendo a plenitude da qualidade de vida do ser humano o horizonte para sua efetivação prática (Ribas, 2019).

As múltiplas dimensões atreladas ao ato de nutrir-se, portanto, colocam o Direito à Alimentação Adequada em destaque. Diante disto, diferentes interesses políticos, econômicos e culturais, como os do agronegócio, da indústria alimentícia e farmacêutica, bem como, da grande mídia e de diversas categorias profissionais influenciam fortemente a temática e necessitam ser atentados.

Neste sentido, conforme Padrão et al. (2017), as intervenções sobre a questão social da alimentação e consequências nutricionais devem ocorrer coordenadamente e intersetorialmente no plano macroestrutural, com medidas que articulem esferas e instâncias de poder, entre elas as ações educativas. Diante destes fatores, acredita-se que um dos caminhos possíveis para a garantia de tal direito seja a educação em saúde.

\section{A Educação em Saúde e a Alimentação}

Desde o século XVIII, Educação e Saúde foram imbricadas como binômios indissociáveis e interdependentes. Em particular nas últimas décadas, o Brasil tem legitimado essa relação por meio das políticas públicas nacionais, pois parte-se da premissa de que é difícil se alcançar bons índices educacionais sem qualidade em saúde e, ao mesmo tempo, a saúde é potencializada por boas práticas de educação básica (Santos \& Santos, 2021). 
Diante deste movimento, fortaleceu-se e oficializou-se a noção de educação em saúde, ou seja, uma concepção que deve guiar práticas de profissionais, gestores e população em geral. Entende-se que a educação em saúde é fundamental para a emergência de melhores índices ligados à qualidade de vida das populações, pois acredita-se que as pessoas precisam ter autonomia para o manejo de cuidados individuais e coletivos (Falkenberg et al, 2014).

Torna-se fundamental que práticas educativas, tanto as escolares quanto as culturais, sejam desempenhadas para o esclarecimento da importância do papel da sociedade em relação à melhoria das condições de saúde e, consequentemente, ao desdobramento de modos de vida mais promissores. Os saberes em saúde, neste contexto, surgem dos debates e disputas entre a ciência e os conhecimentos e práticas populares. A educação em saúde opera na direção de engajar a população na preservação e manutenção da saúde ao mesmo tempo em que problematiza e reorienta práticas de saúde visando torná-las mais significativas e diversificadas (Darsie \& Weber, 2021; Falkenberg et al, 2014).

As práticas educativas devem servir para transformar e superar quaisquer condições de vida que não sejam adequadas às necessidades e à garantia de direitos básicos. Educar acerca da alimentação e nutrição como forma de liberdade deve ser, portanto, entendido como um ato político, encaixado no contexto da educação em saúde. Para tanto, educadores que pretendem contribuir nesse processo devem ser capazes de reconhecer a multidimensionalidade das realidades sociais.

Torna-se importante destacar que a pedagogia tradicional, baseada em resultados quantitativos, por vezes ilude profissionais e estudantes em relação à qualidade dos dados disponíveis e das informações compartilhadas. Essa modalidade pedagógica, por direcionar-se para grupos humanos por meio de protocolos totalizantes, desconsidera o potencial de cada pessoa ao mesmo tempo em que não valoriza a autonomia, a criatividade e a criticidade, enquanto geradoras de conhecimento. Apesar das inovações tecnológicas e didáticas nesse modelo, a história da educação tem mostrado que poucas vezes a pedagogia tradicional confere espaço aos educadores, aprendizes e gestores da educação para refletirem sobre suas práticas.

O ideal, conforme concepções contemporâneas acerca da educação, é que todos os níveis educacionais valorizem e promovam atitudes questionadoras, fortalecendo a liberdade para elaboração de argumentos contraditórios, emergentes por meio da experimentação e experiência coletivas em diferentes cenários de ensino-aprendizagem. (Bruna et al., 1996; Gadotti, 2000). Além da aceitação e do estímulo aos questionamentos e críticas qualificadas, em qualquer ação educativa é preciso que a criação de redes de cooperação, baseadas em relações de confiança e em laços de solidariedade sejam valorizadas, conferindo assim maior chance de legitimação das lutas sociais, arregimentando forças transformadoras e desnudando o cerceamento do debate público (Medeiros \& Marques, 2003).

Em relação ao Direito Humano à Alimentação Adequada, é importante lembrar que a comensalidade - resumida pelo entendimento sobre o que, onde e como alimentar-se e com quem fazê-lo - inclui variadas representações sociais. Para tanto, deve ser complementada com a percepção de comestibilidade, que se caracteriza pelos processos simbólicos, construídos por uma rede de signos e significados culturais e socioeconômicos que entrelaçam o alimento, o comer e o que seja habitualmente comestível para cada indivíduo. Isso diferencia-se de acordo com as formas como cada indivíduo vê e se relaciona com o mundo e com outros indivíduos (Carneiro, 2003; Minnaert, 2013; Montanari, 2013).

Neste sentido, podem ser imaginadas algumas das categorias temáticas que se mostram potentes enquanto referências para o debate transversal sobre a alimentação: cidadania, sustentabilidade, globalização e consequências locais, transdisciplinaridade e holismo, dialogicidade e dialeticidade. Nessa lógica é fundamental lembrar a definição de práxis, cunhada por Paulo Freire, como uma ação associada à reflexão sobre a ação que configura-se enquanto atitude potencialmente transformadora (Chauí, 2004; Nascimento et al., 2017)

Nesta mesma direção, a proposta de Freire $(1992,1996)$ oportuniza a competência da leitura e entendimento do mundo de forma crítica, contribuindo com o desenvolvimento da habilidade de interpretar realidades, incluindo as relações de 
produção e suas alterações geradas pelas mudanças tecnológicas. Permite-se, assim, que ocorram indignações e resistências diante das desigualdades de oportunidades e do desrespeito aos direitos humanos.

As ações supracitadas devem ser balizadas pelos conteúdos extraídos do cotidiano prático, permitindo criar vivências e relações que podem ser fortalecidas por meio de ações de Educação Alimentar e Nutricional (EAN), no Brasil. De forma complementar, a prática em alimentação e nutrição, advinda da qualificação para o apoderamento, deve dialogar com a vida sociocultural, econômica e política do indivíduo ou da comunidade, expondo suas imperfeições.

\section{Educação Alimentar e Nutricional: um Caminho para o Apoderamento}

De acordo com o Marco de Referência de Educação Alimentar e Nutricional para as Políticas Públicas (Brasil, 2012) e conforme Pereira, Moreira e Nunes (2020), a Educação Alimentar e Nutricional (EAN) compreende um conjunto de estratégias que têm como objetivos a promoção de hábitos saudáveis voluntários e a promoção da cultura e valorização da alimentação. Entende-se, neste contexto, que "a prática alimentar saudável é constituída por alimentos de qualidade e de vários tipos, em quantidades suficientes para atender às necessidades nutricionais diárias de cada ser humano, de modo que sejam atingidos os objetivos de saúde, prazer e convívio social" (Pereira et al., 2020, p. 2).

Para além disso, nesta reflexão, argumenta-se que a Educação Alimentar e Nutricional deve ser entendida como uma ferramenta que estimula, por meio dos conhecimentos relacionados à educação em saúde, a participação política e cidadã de indivíduos e populações nas dinâmicas que envolvem a vida. A EAN, portanto, a partir dos preceitos da educação em saúde, deve manejar ações teórico-práticas que visem promover estratégias de politização acerca do acesso e conhecimento acerca de alimentos.

Para tanto, práticas educativas que seguem por esta perspectiva devem considerar debates sobre a territorialidade de resistência, conflitos de terra e seus impactos na insegurança alimentar e nutricional, reforma dos sistemas alimentares e incentivo às práticas sustentáveis e agroecológicas de produção e distribuição de alimentos. Tais ações, quando operadas de forma adequada, podem reverberar na transformação das políticas públicas, impulsionar a criação e o fortalecimento de circuitos curtos de suprimentos alimentares locais resilientes e incentivar a geração de emprego/renda na economia solidária (Brasil, 2012).

É importante que as ações de Educação Alimentar e Nutricional abordem também as estratégias corporativistas do agronegócio, considerando que tal modelo pode ser prejudicial aos sujeitos e ao direito à alimentação, especialmente por se basearem em relações comerciais e econômicas em uma perspectiva neoliberal que, por definição, individualiza problemas de ordem social. As práticas educacionais devem, também, dar visibilidade aos ataques que afetam os biomas brasileiros, desacomodar sujeitos acerca da necessidade de restauração da biodiversidade, problematizar o excesso de consumo de alimentos de origem animal, o aumento da emissão de carbono e estimular o redirecionamento dos subsídios agrícolas para a produção de alimentos in natura ou minimamente processados (Brasil, 2014).

Além disso, conforme sugerido pelo Guia Alimentar para a População Brasileira (Brasil, 2014) a EAN precisa investir em discussões sobre hortas domiciliares, comunitárias e institucionais, bem como, sobre a agricultura urbana e periurbana. Tais movimentos são relevantes para a valorização e para o fortalecimento do Sistema de Segurança Alimentar e Nutricional Brasileiro e da Política Nacional de Soberania e Segurança Alimentar e Nutricional, afinal de contas, conforme discutido anteriormente, trata-se de um direito fundamental.

Partindo de tais problemáticas, pensar em práticas alimentares sustentáveis e responsáveis pode significar uma mudança de paradigmas, por meio da qual o capital deixa de ser central e as populações se tornam os pontos de interesse e agentes transformadores. Deste modo, por meio de parcerias com os movimentos sociais, a Educação Alimentar e Nutricional 
pode, ainda, ser uma ação extensionista de advocacy $^{l}$ no ambiente do poder legislativo e judiciário, visando, por exemplo, contribuir com a correção de distorções tributárias, como ocorre com as bebidas açucaradas e demais alimentos ultraprocessados. Ainda, pode transformar a regulação da publicidade de alimentos e dar visibilidade ao descumprimento das legislações da Agência Nacional de Vigilância Sanitária Brasileira, por parte de alguns setores da cadeia produtiva de alimentos.

A EAN pode capacitar indivíduos e populações visando o apoderamento destes. Conforme apontado por Martins Júnior (2003) e diante da amplitude e da complexidade que envolvem a alimentação, a saúde e a educação a valorização das vivências dos sujeitos e comunidades se torna fundamental, portanto, opera-se, aqui, com o termo apoderamento ${ }^{2}$. Por meio deste termo, a participação social nos processos políticos deixa de ser considerada uma concessão dos poderosos e passa a ser entendida como uma conquista. $\mathrm{O}$ apoderamento não se dá de forma espontânea, mas, pressupõe a percepção individual acerca dos mecanismos do poder na sociedade, gerando mobilização social (Martins Júnior, 2003).

Nesse cenário, contribuir com a familiarização por parte de estudantes, membros de conselhos de participação e controle social, bem como manipuladores de alimentos sobre as leis e regulamentos que protegem a alimentação saudável torna-se uma possibilidade de transformações a partir do apoderamento popular. A EAN pode ser transformadora na vida das crianças, adolescentes e adultos a partir da ideia de autocuidado e de cuidado do outro, tanto em instâncias familiares quanto comunitárias.

A EAN deve ser utilizada como ferramenta lúdica e como instrumento promotor de apoderamento a partir das ideias de "comida de verdade", de "habilidades culinárias" e de outros elementos presentes no Guia Alimentar para a População Brasileira (Brasil, 2014). Suas ações devem promover debates que oportunizem reflexões e movimentos de fortalecimento das potencialidades individuais e coletivas e do reconhecimento dos mecanismos de poder que envolvem a área.

Enfim, devem promover o apoderamento da população no que se refere aos alimentos e ao direito a eles. Neste sentido, identificar políticas públicas, diferenciando-as entre as que funcionam ou não e, ainda, atentar aos seus direcionamentos, de modo a considerar as especificidades das comunidades tradicionais e dos povos originários, permite identificar os principais sujeitos afetados pelas ameaças alimentares e nutricionais, oportunizando destacar políticas e programas prioritários.

\section{Considerações Finais}

Conforme apontado, a garantia de alimentação suficiente e saudável é um direito humano universal. Tal situação dispara a necessidade de reflexões que tensionem as políticas públicas ligadas à alimentação e à nutrição. Para além disso, a discussão envolve práticas educacionais, de modo a compreendê-las como propulsoras de saberes que auxiliam comunidades e/ou populações a atingirem índices de saúde favoráveis.

Uma potente estratégia para tais movimentos, a educação em saúde, emerge enquanto política pública que considera demandas e saberes populares enquanto apoiadores do conhecimento científico. Acredita-se, nesta direção, que práticas educativas que valorizem o pensamento crítico, o reconhecimento de diferentes realidades e a problematização das relações de poder podem ser de extrema importância para a transformação de realidades e superação de desafios e adversidades.

Neste contexto, a Educação Alimentar e Nutricional, como ferramenta de apoderamento, deve considerar propostas didáticas e reflexivas que extrapolem os treinamentos tradicionais, que ampliem os conteúdos programáticos, que utilizem

\footnotetext{
${ }^{1}$ Advocacy deve ser entendida como uma prática política manejada por indivíduos, grupos sociais ou organizações, junto aos setores políticos, com o objetivo de influenciar políticas e recursos públicos.

${ }^{2} \mathrm{O}$ termo de origem inglesa empoderamento - empowerment -, comumente utilizado em discussões como esta, considera a emergência de poder exercido por um grupo minoritário que intenta ou passa a comandar o majoritário. Diante disto, neste texto, optou-se pela ideia de apoderamento, tendo em vista entendimentos individuais sobre os mecanismos de poder.
} 
estratégias educacionais coerentes com a finalidade do apoderamento do ser, permitindo que cada agente possa agir visando transformar o mundo do trabalho, superar as desigualdades existentes na vida humana e transpor os entraves à promoção, proteção, garantia, efetivação e defesa dos direitos humanos.

Entende-se que não contribuir com a promoção e a proteção do direito humano à alimentação adequada, é assumir um posicionamento de apoio à violação desse direito e contrário a um projeto político transformador. Embora defender essa causa não seja simples, é preciso fazer a trajetória da resistência no coletivo, visando persistir nessa disputa de narrativas e de espaços de luta dentro do contexto educacional. Diante de tais argumentos, pretende-se que novas discussões sejam desencadeadas, de modo a estimularem transformações que favoreçam diferentes sujeitos e comunidades.

\section{Referências}

ABESO (2021). Mapa da Obesidade 2008-2009. Disponível em: https://abeso.org.br/obesidade-e-sindrome-metabolica/mapa-da-obesidade/.

Behring, E. R., \& Boschetti, I. (2017). Política social: fundamentos e história. Ed.Cortez.

Brasil. (2012). Marco de referência de educação alimentar e nutricional para as políticas públicas. Ministério do Desenvolvimento Social e Combate à Fome. Brasil. (2014). Guia Alimentar para a População Brasileira. Secretaria de Atenção à Saúde \& Departamento de Atenção Básica.

Bruna, J., Andrade, L. R., \& Strazynski, G. M. R. trad. (1996). Sócrates - Vida e Obra. In Nova Cultural (Ed.), Os Pensadores.

Carneiro, H. S. (2003). Comida e Sociedade: Uma história da alimentação. In História: Questões \& Debates. Elsevier.

Chauí, M. (2004). Convite a filosofia. Ed. Ática.

Darsie, C. \& Weber, D. L. (2021) Geografia da Saúde e Educação Básica: um panorama. In: Paim, R. O.; Pereira, A. M. O.; Copatti, C.; Gengnagel, C. L. (Org.). Geografias que fazemos: educação geográfica em diferentes contextos (Coleção: Percursos de educação geográfica). 1ed.Curitiba-PR: Editora CRV, 2, 189-198.

El País. (2021). Ossos de boi, arroz e feijão quebrado formam o cardápio de um Brasil que empobrece: a pandemia aprofundou ainda mais a situação precária vivida por milhões de brasileiros. https://brasil.elpais.com/brasil/2021-07-25/arroz-quebrado-bandinha-de-feijao-e-ossos-de-boi-vao-para-o-prato-de-umbrasil-que-empobrece.html

Falkenberg, M. B. et al. (2014). Educação em saúde e educação na saúde: conceitos e implicações para a saúde coletiva. Ciência \& Saúde Coletiva, 19(03), 847-852. https://doi.org/10.1590/1413-81232014193.01572013.

FIAN. Organização pelo Direito Humano à Alimentação e à Nutrição Adequadas. Relatório de Monitoramento do Direito à Alimentação e à Nutrição durante a COVID 19 (2020). http://www.fao.org/emergencies/resources/documents/resources-detail/en/c/1312540/

Figueiredo, Adriana do Carmo. (2012). A Arte de Ensaiar com uma perspectiva científica. Palimpsesto, 15, 1-14. https://www.epublicacoes.uerj.br/index.php/palimpsesto/article/view/35239

Freire, P. (1992). Pedagogia da esperança: um reencontro com a pedagogia do oprimido. Ed. Paz e Terra.

Freire, P. (1996). Pedagogia da autonomia: saberes necessários à prática educativa. Ed. Paz e Terra.

G1. (2021) Sem dinheiro para comprar carne, os pernambucanos recorrem a ossos e ovos para fazer as refeições. https://g1.globo.com/pe/pernambuco/noticia/2021/09/02/sem-dinheiro-para-comprar-carne-pernambucanos-recorrem-a-ossos-e-ovos-para-fazer-asrefeicoes.ghtml.

Gadotti, M. (2000). Perspectivas atuais da Educação. Ed. Artes médicas Sul (ed.).

Hugo, V. (2021). Os miseráveis. Trad. Silvana Salermo. Ed. Seguinte.

Leão, M. et al. O Direito Humano à Alimentação Adequada e 0 Sistema Nacional de Segurança Alimentar e Nutricional, 2013. Disponível em: http://www.mds.gov.br/webarquivos/publicacao/seguranca_alimentar/DHAA_SAN.pdf.

Martins Júnior, T. (2003). Apoderamento. SANARE. Revista de Políticas Públicas. https://sanare.emnuvens.com.br/sanare/article/view/111.

Medeiros, A. M. S., \& Marques, M. A. R. B. (2003). Habermas e a teoria do conhecimento. ETD Educação Temática Digital.

Minnaert, A. C. D. S. T. (2013). A comestibilidade e outros mundos possíveis. DEMETRA: Alimentação, Nutrição \& Saúde, 8(0), 265-273. https://doi.org/10.12957/demetra.2013.6172.

Montanari, M. (2013). Comida como cultura. Ed. Senac.

Nascimento, M. N. C. do, Carvalho, M. C. D. V. S., \& Prado, S. D. (2017). Análise sobre orientações políticas de educação alimentar e nutricional. DEMETRA: Alimentação, Nutrição \& Saúde, 12(3). https://doi.org/10.12957/demetra.2017.28669. 
Research, Society and Development, v. 11, n. 1, e14311124629, 2022

(CC BY 4.0) | ISSN 2525-3409 | DOI: http://dx.doi.org/10.33448/rsd-v11i1.24629

ONU/FAO/PMM et al. (2020). Global Network Against Food Crisis. Food Security Information Network. Global Report on Food Crisis. http://www.fao.org/emergencies/resources/documents/resources-detail/en/c/1312540/.

ONU. Declaração Universal dos Direitos Humanos, 1948. 〈https://www.unicef.org/brazil/declaracao-universal-dos-direitos-humanos〉.

Organização Pan-americana de Saúde. Alimentos e bebidas ultraprocessados na América Latina: tendências, efeito na obesidade e implicações para políticas públicas, 2018. https://iris.paho.org/bitstream/handle/10665.2/34918/9789275718643-por.pdf?sequence=5\&isAllowed=y.

Padrão, S. M., Aguiar, O. B., Barão, G. D. O. D. (2017). Educação alimentar e nutricional: A defesa de uma perspectiva hegemônica e histórico-crítica da educação. DEMETRA: Alimentação, Nutrição \& Saúde, 12(3). https://doi.org/10.12957/demetra.2017.28642.

Pereira, T. R., Moreira, B., \& Nunes, R. M. (2020). A importância da educação alimentar e nutricional para alunos de séries iniciais. Lynx, 1(1). https://doi.org/10.34019/2675-4126.2020.v1.25591.

Ribas, L. F. de O. (2019). "Cercamentos" do "Comum" no Direito Humano à Alimentação. Revista de Direito Internacional e Direitos Humanos Da UFRJ INTER, 2(2). https://revistas.ufrj.br/index.php/inter/article/view/28834.

Rother, E. T. (2007). Revisão sistemática X revisão narrativa. Acta Paulista de Enfermagem, 20(2). https://doi.org/10.1590/S0103-21002007000200001.

Santos, M. L. D., \& Santos, M. L. (2021). A educação alimentar e nutricional enquanto estratégia libertadora de promoção de saúde e empoderamento social. Journal of Multiprofessional Health Research, 2(1). http://journalmhr.com/. 